\title{
Analysing Biochemical Parameters and Developing Risk Prediction Models in Patients with Schizophrenia and Bipolar Disorder
}

\author{
Ismail Eren Polat ${ }^{1}$ (D), Dilek Kuzay ${ }^{2^{*}}$ (D), Naime Meric Konar ${ }^{3}$ \\ ${ }^{1}$ Recep Tayyip Erdogan University, Faculty of Medicine, Department of İnternal Medicine, Rize, Turkey \\ ${ }^{2}$ Ahi Evran University, Faculty of Medicine, Department of Physiology, Kirsehir, Turkey \\ ${ }^{3}$ Ahi Evran University, Faculty of Medicine, Department of Biostatistics, Kirsehir, Turkey \\ *Corresponding author: Dilek Kuzay E-mail: dilekkuzay@gmail.com ORCID: 0000-0002-1460-9883 \\ Received: 3 December 2020 Accepted: 14 May 2021
}

\begin{abstract}
Objectives: The aim of this study is to analyze biochemical parameters and develop risk prediction models in patients with schizophrenia and bipolar disorder.

Methods: Biochemical values of 65 schizophrenia and 65 bipolar disorder patients were statistically compared by analyzing thyroid function tests (TSH, T3 and T4), 25-0H vitamin D3, calcium, ferritin, iron, folate, vitamin B12, and magnesium levels. Risk analysis of the control group was evaluated in line with the obtained findings.

Results: Male patients diagnosed with schizophrenia were observed to have lower T3 level than that of control group $(p<0.001)$. Vitamin B12 value and Ferritin levels of male patients diagnosed with bipolar disorder were higher than those of control group $(p<0.05)$, while their T3 levels were lower comparing to control group $(p<0.001)$. Female patients diagnosed with schizophrenia were observed to have higher Ferritin levels $(p<0.05)$ and lower T3 levels comparing to control group $(p<0.001)$. When female patients diagnosed with bipolar disorder were compared with control group, their Ferritin levels and T4 levels were observed to be higher $(p<0.05)$.

Conclusion: Unless an intervention is made by detecting vitamin deficiency, the treatment may fail with psychotropic medications. In psychotic symptoms, in addition to antipsychotic treatment, replacement of low serum vitamin level may have positive effects on recovery. Therefore, serum vitamin B12, T3, T4 and TSH hormones, and ferritin levels can be recommended to be used as routine screening tests especially in the first applications of psychiatric patients with cognitive disorders.
\end{abstract}

Keywords: schizophrenia, bipolar disorder, vitamin D, thyroxine, vitamins, minerals

\section{INTRODUCTION}

Schizophrenia is a psychiatric disorder involving chronic or recurrent psychosis. It is generally related to disorders in social or professional functionality [1]. It is ranked as one of the top ten illnesses that contribute to the global burden of disease and defined as one of the most disabling and economically catastrophic medical disorders by the World

○ 2021 by the authors; licensee EJMETS by Bastas, UK. This article is an open access article distributed under the terms and conditions of the Creative Commons Attribution License (http://creativecommons.org/licenses/by/4.0/). 
Health Organization [2]. Bipolar disorder is a mood disorder characterized with mania attacks, hypomania, and major depression [1].

When several studies are investigated, schizophrenia and bipolar disorder are seen to be related with certain biochemical parameters. To illustrate, psychosis, behavioral disorder, and depression may accompany hypothyroidism; anxiety, hyperactivity, labile personality, mania, temporary psychosis may rather accompany hyperthyroidism [3].

Thyroid hormone has a vital role in the maturation and function of central nervous system. Thyroid function disorder does not cause a structural damage in the brain, but may cause a variety of psychiatric disorders [4].

Since schizophrenia is commonly observed in people born in winter, this gives rise to thought of its relationship with vitamin D [5]. In a study considering calcium value, significantly calcium level in depression patient and bone mineral density was found to be significantly lower comparing to control group [6]. In another case report, it was mentioned that unless $\mathrm{Ca}$ and $\mathrm{Mg}$ are normalized in a patient with psychotic disorder, the treatment cannot be successful [7].

There are a number of studies indicating that low Folate evels decrease the response to the treatment carried out with antidepressants. In a study conducted with hospitalised psychiatric patients, it was shown that the frequency of low serum vitamin B12 level was 5-30\% while it was 3-5\% in the group with no psychiatric problem [8].

This study comparatively analyzes thyroid function tests (TSH, T3 and T4), 25-0H vitamin D3, calcium, ferritin, iron, Folate vitamin B12, and magnesium levels of 65 schizophrenia and 65 bipolar disorder patients who applied to psychiatry clinic in the last 5 years with those of control group. Then with the obtained results, the study aims to develop risk prediction models to determine the risk of schizophrenia and bipolar disorder in male and female patients separately and to investigate the correlation related with these illnesses.

\section{METHOD}

Biochemical values of 65 bipolar and 65 schizophrenics applied to the psychiatry clinic in the last 5 years and 65 people in control group who applied to internal diseases clinic without any psychiatric diagnosis were retrospectively analyzed. All procedures were approved by the Ethics Committee (Project number 2018-14/121).
Those without any metabolic diseases that could affect their $\mathrm{TSH}, \mathrm{T} 3, \mathrm{~T} 4,25-\mathrm{OH}$ D3, calcium, ferritin, iron, Folate and magnesium levels and those who were not on medication that could affect the levels of these parameters were selected for the control group.

Biochemical analyses were conducted in biochemistry laboratory of Ahi Evran University Hospital on 'Cobas C 8000 Autoanalyser, Roche Dragnostics, Manheim Germany' device by using kit.

Also, risk prediction models were developed in this study to determine the risk of schizophrenia and bipolar disorder in male and female patients separately with multinomial logistics regression model. In the interpretation of how effective the markers are in the diagnosis of schizophrenia and bipolar disorder, Odds ratios and 95\% confidence intervals for Odds ratios obtained from the regression model were utilized.

\section{Statistical Analysis}

In order to compare schizophrenia group, bipolar group, and control group in terms of biochemical markers, one way analysis of variance (ANOVA) was used when normality assumption were confirmed and the Kruskal-Wallis test was used when not confirmed. When there was a statistically significant difference among the groups, Bonferroni test or Dunn test were used as post-hoc test in order to determine the group causing the difference. As basic descriptive statistics, when observations had a normal distribution, mean-standard deviation values were given; when they did not have a normal distribution, median, minimummaximum values were given. In the determination of the relationship between individual's being diagnosed with schizophrenia and bipolar disorder and biochemical markers, multinomial logistic regression model, which is used when dependent variable has three or more groups (nominal), was employed. Risk prediction models were developed to determine the risk of schizophrenia and bipolar disorder in male and female patients separately with this multinomial logistic regression model. In the interpretation of how effective the markers are in the diagnosis of schizophrenia and bipolar disorder, Odds ratios and $95 \%$ confidence intervals for Odds ratios obtained from the regression model were utilized. In all statistical analyses, $p<0.05$ was accepted as the statistical significance in all analyses. All the analyses were conducted with SPSS 22.0 (IBM Corp. Released 2013. IBM SPSS Statistics for Windows, Version 22.0. Armonk, NY: IBM Corp.). 
Table 1. Biochemical Parameter Levels in Control, Schizophrenia and Bipolar Disorder Groups (Males)

\begin{tabular}{|c|c|c|c|c|}
\hline Indicator tests & Control & Schizophrenia & Bipolar & p-value \\
\hline TSH (mUl/L) & $2.145[0.83-27.20]$ & $2.335[0.57-13.43]$ & $2.35[0.30-4.830]$ & $0.689^{*}$ \\
\hline Vitamin B12 (pg/ml) & $253.300 \pm 76.233$ & $254.069 \pm 154.012$ & $374.524 \pm 305.414$ & $0.013^{* *}$ \\
\hline Ferritin (mg/dl) & $119.820 \pm 53.144$ & $108.884 \pm 81.290$ & $177.516 \pm 110.859$ & $0.035^{* *}$ \\
\hline Vitamin D (ng/dl) & $21.840 \pm 7.784$ & $19.389 \pm 12.818$ & $20.058 \pm 7.419$ & $0.470^{* *}$ \\
\hline Folate (ng/ml) & $7.562 \pm 1.915$ & $6.511 \pm 2.703$ & $6.816 \pm 2.160$ & $0.090^{* *}$ \\
\hline Ca (mg/dl) & $9.419 \pm 0.293$ & $9.547 \pm 0.423$ & $9.405 \pm 0.486$ & $0.239^{* *}$ \\
\hline Fe (ug/dl) & $95.5[24-212]$ & $92.5[11-404]$ & $81[48-160]$ & $0.306^{*}$ \\
\hline Mg (mg/dl) & $2.1[1.7-2.5]$ & $2.1[1.8-2.4]$ & $2[1.8-2.4]$ & $0.324^{*}$ \\
\hline T3 (ng/ml) & $3.469 \pm 0.525$ & $2.969 \pm 0.514$ & $3.154 \pm 0.491$ & $<0.001^{* *}$ \\
\hline T4 (ng/dl) & $1.261 \pm 0.150$ & $1.321 \pm 0.209$ & $1.351 \pm 0.191$ & $0.097^{* *}$ \\
\hline
\end{tabular}

*: Kruskal-Wallis Test

**: One-Way Analysis of Variance (ANOVA)

\section{RESULTS}

According to results, the mean age of male patients who applied to the psychiatry clinic in the last 5 years and were diagnosed with schizophrenia is $39.0 \pm 13.9$; the mean age of those with bipolar disorder diagnosis is $42.6 \pm 10.3$. Of those diagnosed with schizophrenia in the last 5 years, $55.4 \%$ are male and of those diagnosed with bipolar disorder, $61.5 \%$ are female.

$\mathrm{T}_{3}$ level of male patients with schizophrenia $(2.9 \pm 0.5 \mathrm{ng} / \mathrm{ml})$ and bipolar diorder diagnosis $2.881 \pm 0.595 \mathrm{ng} / \mathrm{ml}$ ) were seen to be lower than that of control group (3.469 \pm 0.525 $\mathrm{ng} / \mathrm{ml})(\mathrm{p}<0.001)$. Furthermore, bipolar diagnosis group was found to have significantly higher Ferritin (177.516 \pm $110.859 \mathrm{ng} / \mathrm{ml})$ and Vitamin B12 (374.524 $\pm 305.414 \mathrm{ng} / \mathrm{ml})$ levels than that of control and schizophrenia diagnosis group ( $p=0.035$ for Ferritin and $p=0.013$ for Vitamin B12, respectively). No statistically significant difference was found in their TSH, 25-OH D3, Folate, $\mathrm{Ca}, \mathrm{Fe}, \mathrm{Mg}, \mathrm{T}_{4}$ levels (p>0.05).

Ferritin levels of females diagnosed with schizophrenia (45 $\mathrm{mg} / \mathrm{dl}$ ) and bipolar disorder (49 $\mathrm{mg} / \mathrm{dl})$ were higher than that of control group ( $25 \mathrm{mg} / \mathrm{dl})(\mathrm{p}=0.002)$; however, T3 levels of schizophrenia $(2.707 \pm 0.443)$ were lower compared to control (3.114 \pm 0.389$)$ and bipolar (2.881 \pm 0.595$)$ group $(p<0.001)$. Moreover, T4 levels of bipolar group (1.286 \pm 0.270 ) were found to be significantly higher than control group (1.166 \pm 0.158$)$ ( $p$-value from Bonferroni post-hoc test for these two groups is 0.037$)$; however, bipolar (1.286 \pm 0.270 ) and schizophrenia-diagnosed groups (1.268 \pm 0.239$)$ ( $p$-value from Bonferroni post-hoc test for these two groups is 0.999$)$ and control $(1.166 \pm 0.158)$ and schizophrenic groups $(1.268 \pm 0.239)$ were observed to have similar T4 levels ( $p$-value from Bonferroni post-hoc test for these two groups is 0.137 ). Overall, female subjects were seemed to have statistically different T4 levels ( $p=0.026$ for One-Way ANOVA). No statistically significant difference was found in their TSH, Vitamin B12, 25-OH D3, Folate, Ca, Fe, and Mg levels ( $p>0.05)$.

Results of the comparison of biochemical parameter levels in Control, Schizophrenic and Bipolar disorder groups are presented in Table $\mathbf{1}$ and Table $\mathbf{2}$ for male and female patients separately.

According to the results of logistics regression analysis, 1 unit ( $\mathrm{ml} / \mathrm{ng}$ ) increase in Ferritin value increases the risk of bipolar diagnosis by 1 in males comparing to healthy individuals and 1 unit $(\mathrm{pg} / \mathrm{ml})$ of increase in B12 increases the risk by $6 \%$ o. 1 unit (ng/dl ) increase in T4 value increases the risk of bipolar by $6.7 \%$ and schizophrenia by $4.8 \%$. However, 1 unit (ng/ml) increase in T3 value decreases the risk of bipolar diagnosis by $2.1 \%$ and schizophrenia by $2.4 \%$. (The risk of being bipolar and schizophrenic is low for males with high T3 level; the risk of bipolar diagnosis is high for males with high Ferritin and vitamin B12; the risk of being both bipolar and schizophrenic is high for males with high T4 value). Results are presented in Table 3.

According to the results of logistics regression analysis, 1 unit ( $\mathrm{ng} / \mathrm{mL}$ ) increase in T4 value increases the risk of bipolar diagnosis by $4.6 \%$ and schizophrenia by $4.9 \%$ in females. However, 1 unit (ng/ml) increase in T3 value decreases the risk of bipolar diagnosis by $1.6 \%$ and schizophrenia by $2.3 \%$. 1 unit $(\mathrm{mg} / \mathrm{dl})$ increase in Ferritin value decreases the risk of bipolar diagnosis by $4.5 \%$ and schizophrenia by $2.9 \%$. (The risk of being bipolar and schizophrenic is low for females with high T3 level; the risk of schizophrenia and bipolar diagnosis is low for females with high Ferritin value; the risk of being both bipolar and schizophrenic is high for females with high T4 value). Results are presented in Table 4. 
Polat et al. / Analysing Biochemical Parameters in Patients with Schizophrenia and Bipolar Disorder

Table 2. Biochemical Parameter Levels in Control, Schizophrenia and Bipolar Disorder Groups (Females)

\begin{tabular}{|c|c|c|c|c|}
\hline Indicator tests & Control & Schizophrenia & Bipolar Disorder & p-value \\
\hline TSH & $2.105[0.960-4.40]$ & $2.25[0.05-7.480]$ & $3[0-9.470]$ & $0.621^{*}$ \\
\hline Vitamin B12 & $261.500[100-740]$ & $296[74-648]$ & $269[110-688]$ & $0.318^{*}$ \\
\hline Ferritin & $25[3-84]$ & $45[2-200]$ & $49[5-198]$ & $0.002^{*}$ \\
\hline Vitamin D & $22.580 \pm 8.415$ & $18.210 \pm 11.198$ & $17.875 \pm 15.173$ & $0.115^{* *}$ \\
\hline Folate & $8.295 \pm 2.250$ & $9.118 \pm 3.399$ & $8.331 \pm 3.117$ & $0.420^{* *}$ \\
\hline Ca & $9.240 \pm 0.359$ & $9.286 \pm 0.404$ & $9.400 \pm 0.492$ & $0.196^{* *}$ \\
\hline Fe & $74.980 \pm 34.416$ & $69.931 \pm 32.556$ & $67.800 \pm 33.439$ & $0.585^{* *}$ \\
\hline Mg & $2.020 \pm 0.212$ & $2.092 \pm 0.192$ & $2.044 \pm 0.197$ & $0.313^{* *}$ \\
\hline T3 & $3.114 \pm 0.389$ & $2.707 \pm 0.433$ & $2.881 \pm 0.595$ & $<0.001^{* *}$ \\
\hline T4 & $1.166 \pm 0.158$ & $1.268 \pm 0.239$ & $1.286 \pm 0.270$ & $0.026^{* *}$ \\
\hline
\end{tabular}

*: Kruskal-Wallis Test

**: One-Way Analysis of Variance (ANOVA)

Table 3. Logistic regression analysis results for males

\begin{tabular}{|c|c|c|c|c|c|c|}
\hline \multirow{9}{*}{ Bipolar } & \multirow{2}{*}{ Indicator } & \multirow{2}{*}{$\boldsymbol{\beta}$} & \multirow{2}{*}{ p-value } & \multirow{2}{*}{ Odds Ratio (OR) } & \multicolumn{2}{|c|}{ 95\% Confidence Interval } \\
\hline & & & & & Lower Limit & Upper Limit \\
\hline & ViT.B12 & 0.006 & 0.012 & 1.006 & 1.001 & 1.011 \\
\hline & FERRITIN & 0.01 & 0.006 & 1.010 & 1.003 & 1.018 \\
\hline & AGE & 0.037 & 0.267 & 1.037 & 0.972 & 1.106 \\
\hline & FOLATE & -0.002 & 0.191 & 0.998 & 0.996 & 1.001 \\
\hline & T3 & -0.021 & 0.007 & 0.979 & 0.964 & 0.994 \\
\hline & T4 & 0.067 & 0.001 & 1.069 & 1.028 & 1.112 \\
\hline & Constant & -5.815 & 0.144 & & & \\
\hline \multirow{9}{*}{ Schizophrenia } & \multirow{2}{*}{ Indicator } & \multirow{2}{*}{$\boldsymbol{\beta}$} & \multirow{2}{*}{ p-value } & \multirow{2}{*}{ Odds Ratio (OR) } & \multicolumn{2}{|c|}{ 95\% Confidence Interval } \\
\hline & & & & & Lower Limit & Upper Limit \\
\hline & Vіт.B12 & 0.002 & 0.351 & 1.002 & 0.998 & 1.007 \\
\hline & FERRITIN & 0.001 & 0.853 & 1.001 & 0.993 & 1.008 \\
\hline & AGE & 0.055 & 0.054 & 1.057 & 0.999 & 1.118 \\
\hline & FOLATE & -0.002 & 0.055 & 0.998 & 0.995 & 1.000 \\
\hline & T3 & -0.024 & 0.001 & 0.977 & 0.963 & 0.99 \\
\hline & T4 & 0.048 & 0.004 & 1.05 & 1.015 & 1.085 \\
\hline & Constant & -0.053 & 0.007 & & & \\
\hline
\end{tabular}

Table 4. Logistic regression analysis results for females

\begin{tabular}{|c|c|c|c|c|c|c|}
\hline \multirow{9}{*}{ Bipolar Disorder } & \multirow{2}{*}{ Indicator test } & \multirow{2}{*}{$\beta$} & \multirow{2}{*}{ p-value } & \multirow{2}{*}{ Odds Ratio (OR) } & \multicolumn{2}{|c|}{ 95\% Confidence Interval } \\
\hline & & & & & Lower Limit & Upper Limit \\
\hline & Viт.B12 & 0.016 & 0.08 & 1.016 & 0.998 & 1.034 \\
\hline & FERRITIN & -0.045 & 0.044 & 0.956 & 0.915 & 0.999 \\
\hline & AGE & 0.014 & 0.559 & 1.014 & 0.968 & 1.061 \\
\hline & FOLATE & 0.012 & 0.085 & 1.012 & 0.998 & 1.026 \\
\hline & T3 & -0.016 & 0.023 & 0.984 & 0.971 & 0.998 \\
\hline & T4 & 0.046 & 0.005 & 1.048 & 1.014 & 1.082 \\
\hline & Constant & -12.372 & 0.054 & & & \\
\hline \multirow{9}{*}{ Schizophrenia } & \multirow{2}{*}{ Indicator test } & \multirow{2}{*}{$\boldsymbol{\beta}$} & \multirow{2}{*}{ p-value } & \multirow{2}{*}{ Odds Ratio (OR) } & \multicolumn{2}{|c|}{ 95\% Confidence Interval } \\
\hline & & & & & Lower Limit & Upper Limit \\
\hline & Viт.B12 & 0.016 & 0.089 & 1.016 & 0.998 & 1.035 \\
\hline & FERRITIN & -0.029 & 0.228 & 0.971 & 0.926 & 1.019 \\
\hline & AGE & 0.026 & 0.306 & 1.027 & 0.976 & 1.08 \\
\hline & FOLATE & 0.005 & 0.541 & 1.005 & 0.99 & 1.019 \\
\hline & T3 & -0.023 & 0.002 & 0.977 & 0.963 & 0.992 \\
\hline & T4 & 0.049 & 0.004 & 1.05 & 1.016 & 1.086 \\
\hline & Constant & -5.036 & 0.461 & & & \\
\hline
\end{tabular}




\section{Logistics Regression Model (Mathematical Equation)}

Multinomial logistics regression models were developed to predict the risk of schizophrenia and bipolar disorder in individuals separately for males and females. With the help of these models, it is possible to predict the risk of schizophrenia or bipolar disorder by placing the biochemical marker values and ages on the equation. Multinomial logistic regression equations obtained for males and females separately are given below:

$\Pi_{\text {Bipolar }}=$

$e^{\left(-5.815+0.006 * \text { VitaminB } 12+0.01 * \text { Ferritin }+0.037 * A g e-0.02 * \text { FOLATE }-0.021 * T_{3}+0.067 * T_{4}\right)}$ $\overline{1+e^{\left(-5.815+0.006 * \text { VitaminB } 12+0.01 * \text { Ferritin }+0.037 * \text { Age }-0.02 * \text { FOLATE }-0.021 * T_{3}+0.067 * T_{4}\right)}}$

$\pi_{\text {Schizophrenic }}=$

$e^{\left(-0.053+0.002 * \text { VitaminB } 12+0.001 * \text { Ferritin }+0.055 * A g e-0.002 * \text { FOLATE }-0.024 * T_{3}+0.048 * T_{4}\right)}$ $\overline{1+e^{\left(-0.053+0.002 * V \text { itaminB } 12+0.001 * \text { Ferritin }+0.055 * A g e-0.002 * \text { FOLATE }-0.024 * T_{3}+0.048 * T_{4}\right)}}$ e : Natural logarithm (2.71)

\section{Equation 1: Logistics Regression Model in Males}

$\Pi_{\text {Bipolar }}=$

$e^{\left(-12.372+0.016 * \text { Ferritin }-0.045 * \text { Vitamin } 25 O H+0.014 * \text { Age }+0.012 * C a-0.016 * T_{3}+0.046 * T_{4}\right)}$ $1+e^{\left(-12.372+0.016 * \text { Ferritin }-0.045 * \text { Vitamin } 25 O H+0.014 * A g e+0.012 * C a-0.016 * T_{3}+0.046 * T_{4}\right)}$

$\Pi_{\text {Schizophrenic }}=$

$e^{\left(-5.036+0.016 * \text { Ferritin }-0.029 * \text { Vitamin } 25 O H+0.026 * \text { Age }+0.005 * C a-0.023 * T_{3}+0.049 * T_{4}\right)}$ $\overline{1+e^{\left(5.036+0.016 * \text { Ferritin }-0.029 * \text { Vitamin } 25 O H+0.026 * A g e+0.005 * C a-0.023 * T_{3}+0.049 * T_{4}\right)}}$

e : Natural logarithm (2.71)

\section{DISCUSSION}

The aim of this study is to separately analyze thyroid function tests (TSH, T3, and T4), 25-0H D3, $\mathrm{Ca}^{2+}$, ferritin, iron, Folate, vitamin B12, and magnesium levels of 65 schizophrenia and 65 bipolar disorder patients who applied to psychiatry clinic in the last 5 years with those of control group. Then with the obtained results, the study aims to develop risk prediction models to determine the risk of schizophrenia and bipolar disorder in male and female patients separately.

According to the results, T3 hormone level in male patients diagnosed with both bipolar disorder and schizophrenia was seen to be lower than that of control group, and no difference was observed in TSH level. T3 hormone level in female patients diagnosed with schizophrenia was seen to be lower than that of control group, and no difference was observed in TSH level. T4 hormone level was found to be high in female patients with bipolar disorder diagnosis.

The results obtained by Schrami et al. are consistent with our results. They reported that psychosis, behavioral disorder, and depression may accompany hypothyroidism; anxiety, hyperactivity, labile personality, mania, temporary psychosis may rather accompany hyperthyroidism [13]. In another study, it was reported that the mechanism beneath the relationship between psychiatric symptoms and thyroid function disorders is uncertain; however, the reason of the symptoms could be hyperactivity in hypothalamic-pituitaryadrenal axis. Besides, it was also claimed that improvement in thyroid function disorder may not always result in psychiatric improvement [9]. There is an argument obtained by the results that the number of thyroid function disorder is higher in patients applied to psychiatry clinic comparing to healthy population [10]. Therefore, thyroid function tests are obtained from almost all patients who apply to hospitals with recent diagnosis of depression. However, there is still no clear evidence on the relationship between thyroid function disorder and depression [11].

According to the results of the present study, no difference was observed in vitamin D and calcium levels of both male and female patients with bipolar disorder and schizophrenia comparing to control group.

However, previous studies stated that schizophrenia is more commonly observed in those born in winter as brain development of fetus is negatively affected by the mother's vitamin $D$ deficiency. Though such information is still inconclusive, it draws attention to the development of schizophrenia. For instance, it is known that there is a relationship between schizophrenia and high rate of births in winter, the increase in the frequency of schizophrenia among dark-skinned immigrants moving towards cold climates, prevalence of schizophrenia among those born in cities, and poor nutrition in mother's womb (5).

Schneider et al. investigated 25-OH-D3, 1,25- dihydroxy vitamin D3 $(1,25(\mathrm{OH}) 2 \mathrm{D})$, calcium, phosphate, and PTH levels in 89 patients ( $38 \%$ female) including 34 patients diagnosed with schizophrenia, 30 with alcoholism, and 25 with major depression (MD) and a control group of 31 healthy individuals. In MD and schizophrenia groups, $1,25(\mathrm{OH}) 2 \mathrm{D}$ levels were found to be lower than that of alcoholics and control group. Also, they determined $25-\mathrm{OH}$ D3 level of schizophrenics lower than that of control group. In the study, it was stated that poor quality and under nourishment and lack of sun exposure can be important variables in psychiatric patients [12].

Ang et al. concluded that unless $\mathrm{Ca}^{2+}$ and $\mathrm{Mg}$ are normalized in patients with psychological problems, the treatment cannot be succeeded in the existence of hypocalcemia, hypercalcemia and hypomagnesemia [7].

Levine and Gaoni detected hypoparathyroidism in a 15year-old inpatient with a psychological problem [13]. In a 
study that screened 1566 individuals over the age of 65 , depression scale scores of patients with low bone mineral density (BMD) were found to be high [14].

Schweiger et al. found that spinal BMD was lower in 80 inpatients who were above 40 years of age and had depression comparing to control group [15].

Michelson et al. who investigated the relationship between BMD and depression compared 24 patients with previous or ongoing depression with control group and measured BMD in 3 parts. They found that BMD was lower in all localizations of these patients comparing to control group [6].

Joborn et al. observed frequent anxiety, depression, and cognitive complaint in patients with primary hyperparathyroidism and mild hypercalcemia and detected improvement in mental health a year after parathyroid surgery [16].

A study reported that osteoporosis causes certain psychiatric problems and if not treated, it limits the effectiveness of treatment approaches in psychiatric problems $[17,18]$. There are also studies reporting that depressive symptoms in women with osteoporosis are significantly low; for instance, symptoms including sleep disorder, loss of appetite, fatigue, social anxiety disorder and fear of death have been found $[19,20]$.

The results of the current study showed that vitamin B12 level was high in male patients with bipolar disorder. As for schizophrenia patients, B12 levels were normal in males. B12 levels of female patients were found to be normal.

According to the results of another study, though not frequently observed, psychological symptoms, such as negative thoughts, orientation and concentration disorder, memory decrease, low attention, social indifference, insomnia, personality changes, hallucination, propensity for violence, and paranoia can be seen as a result of low levels of B12. Also, disorders, such as depression, bipolar disorder, psychosis, phobia, chronic fatigue can be seen. In another study conducted with 54 patients, depression with psychotic features was found due to low levels of B12 [21]. In a study carried out with psychiatric inpatients, it was stated that the frequency of low serum B12 level was 5-30\% and $3-5 \%$ among those without any psychiatric problems [8]. It was found that cobalamin levels are below normal in $30 \%$ of schizophrenic cases as well as schizoaffective and organic disorders [22].

In a case report by Danki et al., it was stated that vitamin B12 level was low in a patient hospitalized in psychiatry clinic through emergency with pre-diagnosis of psychotic disorder, and in the next couple of days after the second day when vitamin B12 and Keatrapin were administered, a dramatic improvement was observed in psychotic symptoms, retardation, and cooperation of the patient. They reported that when vitamin B12 administration was carried on, a significant improvement was seen in psychiatry scales and B12 levels after 16 days [21]. These data have a fostering role in the relationship between vitamin B12 and psychiatric illnesses.

Results of the present study showed that Folate levels of males and females in both schizophrenia group and bipolar disorder group were on the same levels with those in control group.

In a study conducted with psychiatry inpatients, low Folate level was $10-33 \%$ while it was $5-8 \%$ in the population without any psychiatric problems [22].

In Corney et al.'s study carried out with 243 patients applied to psychiatry clinic, Folate levels in the blood was screened and it was observed that deficiency in Folate levels was higher in endogen (29\%) and neurotic (11\%) depression [23].

A study was conducted in the USA with 213 outpatients with depression and it was shown that those with low Folate levels had higher depression and responded less to the treatment with standard antidepressants [24].

A number of studies in the literature have shown that low Folate level decreases the response to the treatment with antidepressant. One of those studies concluded that $45 \%$ of patients with normal Folate level responded to the treatment while it was only $7 \%$ in those with low Folate level [23].

In a study by Analan et al., a significant difference was found between score drops of patients with low serum Folate level and patients with normal Folate level in Hamilton Depression Rating Scale at the end of the $42^{\text {nd }}$ day. The same study reported that adding folic acid to the treatment increases the response to the treatment, accelerates the treatment and contributes to the decline of the symptoms of depression [25].

Vitamin B12 and folic acid are closely connected vitamins which play an essential role in certain metabolic activities in central nervous system [23]. Both vitamins are necessary for the conversion of homocysteine into methionine and the production of S- Adenosyl methionine (SAM) [22, 26, 27]. B12 and folic acid are cofactors in the metabolism of 
homocysteine [28]. A number of previous research has linked high blood levels of homocysteine with various psychiatric and neurodegenerative diseases including dementia, depression, schizophrenia, Alzheimer and Parkinson's disease $[23,26]$.

One of these studies conducted by Regland et al. investigated homocysteine levels of 20 untreated schizophrenia patients and found that $9 \%$ of the patients had high homocysteine level [21].

In the present study, it was found that Mg levels of males and females in both schizophrenia and bipolar disorder group were the same with the control group.

There are limited number of studies in the literature linking psychiatric disorders with magnesium. A case report claimed that depressive condition and paresthesia of a 69year-old female patient with Gitelman Syndrome dramatically improved with intravenous magnesium sulfate treatment [29]. Ang et al. reported that a patient showed psychological problems in cases of high-low calcium and low magnesium and the treatment could not be succeeded unless calcium and magnesium were normalized [7].

Results of the present study showed that Ferritin levels are high in male patients with bipolar disorder, female patients with bipolar disorder, and female patients diagnosed with schizophrenia. Iron levels of males and females in both bipolar disorder group and schizophrenia group were in the same levels with control group. A study in the literature investigating the relationship between iron levels and psychiatric disorders reported that patients with bipolar disorder had high plasma iron levels and these people were resistant to treatment. Especially in treatment resistant patients, the necessity of routine screening of iron levels has been emphasized [30].

Limitations of the study; Not working with a homogeneous group, the clinical presentation of the patients, duration of illness, the drugs (antipsychotic, antidepressant, mood stabilizer) they used were not taken into account, selection of cases from a single center, physical diseases of patients and control group, failure to examine treatment histories, confounding factors such as smoking, alcohol, substance use, nutrition and exercise status are not excluded etc.

Some of the strengths of our study are: studying many biochemical parameters with our study, comparison of schizophrenia and bipolar diseases seen as a social health problem under a single study, uncovering the risk prediction models, evaluation of men and women separately, and it has been compared with various studies.

\section{CONCLUSION}

Vitamin deficiencies may lead to the development of psychiatric diseases or may intensify preexisting diseases.

Though they can be reversible at the beginning, especially degenerations developing based on vitamin deficiencies may not be reversed if not treated. Besides, if vitamin deficiencies are not overcome, duration and success of the treatment can be negatively affected. We are positive that, in addition to antipsychotic treatment, replacement of low plasma level vitamin in psychotic symptoms will have positive effects on the recovery.

Thus, blood tests to be demanded for vitamin deficiencies are of high importance in terms of early diagnosis, treatment and prognosis.

As a result, B12 level, ferritin levels, and T3, T4, and TSH hormones can be recommended to be used as routine screening tests in the first applications of patients with cognitive disorders.

Author contributions: All authors were involved in concept, design, collection of data, interpretation, writing, and critically revising the article. All authors approve final version of the article.

Funding: The authors received no financial support for the research and/or authorship of this article.

Declaration of interest: Authors declare no competing interest.

Data availability: Data generated or analysed during this study are available from the authors on request.

\section{REFERENCES}

1. American Psychiatric Association. Diagnostic and Statistical Manual of Mental Disorders, Fifth Edition (DSM-5), American Psychiatric Association, Arlington, VA 2013. (doi: 10.1176/appi.books.9780890425596).

2. Murray CJL, Lopez AD. The Global Burden of Disease, Harvard University Press, Cambridge, MA 1996:21.

3. Rai V, Yadav U, Kumar P, Yadav SK, Gupta S. MethylenetetrahydroFolate reductase A1298C genetic variant\& risk of schizophrenia: A meta-analysis. Indian J Med Res. 2017;145(4):437-47.

4. Tang R, Wang J, Yang L, Ding X, Zhong Y, Pan J, et al. Front. Subclinical Hypothyroidism and Depression: A Systematic Review and Meta-Analysis. Endocrinol (Lausanne). 2019;10:340. (doi: 10.3389/fendo.2019. 00340).

5. Eyles DW, Trzaskowski M, Vinkhuyzen AAE, Mattheisen M, Meier S, Gooch $H$, et al. The association between neonatal vitamin $D$ status and risk of schizophrenia. Sci Rep. 2018;8:17692. (doi: 10.1038/s41598-018-35418-z). 
6. Jiaming Zhou, Yuan Xue. Depression and Bone Mineral Density. J Bone Miner Res. 2020;35(4):821. (doi: 10.1002/jbmr.3965).

7. Amaral AD, Novais C, Coelho MA, Silva A, Curral R, Brandão I, et al. Organic Psychosis Due to Hypoparathyroidism in an Older Adult: A Case Report. Braz J Psychiatry. 2016;38(4):349-50. (doi: 10.1590/15164446-2015-1869).

8. Bhat RS, Chiu E, Jeste DV. Nutrition and geriatric psychiatry: a neglected field. Curr Opin Psychiatry 2005;18(6):609-14. (doi: 10.1097/01.yco.0000186814. 08826.db).

9. Cohen-Woods S, Craig I, Gaysina D, Gray J, Gunasinghe C, Craddock N, et al. The Bipolar Association Case-Control Study (BACCS) and meta-analysis: No association with the 5,10 MethylenetetrahydroFolate reductase gene and bipolar disorder. Am J Med Genet B Neuropsychiatr Genet. 2010;153B(7):1298-304. (doi: 10.1002/ajmg.b. 31101).

10. Analan E, Dogan O, Akyuz G. The role of folic acid in the treatment of major depressive disorder. Anatolian Journal of Psychiatry. 2000;1(1):5-12.

11. Solak Görmüş IZ, Ergene N. Clinical Importance of Magnesium, Genel Tıp Derg. 2003;12(12):69-75.

12. Vitamin B12 deficiency diagnosis and treatment guidelines. Turkish Society of Hematology.National. Treatment Guideline 2011.

13. Schrami FV, Goslar PW, Baxter L, Beason-Held LL. Thyroid stimulating hormone and cognition during severe, transient hypothyroidism. Neuro Endocrinol Lett 2011;32(3):279-85.

14. Tichomirowa MA, Keck ME, Schneider HJ, PaezPereda M, Renner U, Holsboer F, et al. Endocrine disturbances in depression. J Endocrinol Invest. 2005;28(1):89-99. (doi: 10.1007/BF03345535).

15. Engum A, Bjøro T, Mykletun A, Dahl AA. An association between de-pression, anxiety and thyroid function-a clinical fact or an artefact? Acta Psychiatr Scand. 2002;106(1):27-34. (doi: 10.1034/j.1600-0447.2002. 01250.x).

16. Chauhan N, Padhy SK, Shah R, Malhotra S. Vitamin D Deficiency in Children With Psychiatric Illness in a Tertiary Care Hospital in North India. Neurosci Rural Pract. 2019;10(1):16-20. (doi: 10.4103/jnrp.jnrp_169_18).
17. Song L, Luo X, Jiang Q, Chen Z, Zhou L, Wang D, et al. Vitamin D Supplementation is Beneficial for Children with Autism Spectrum Disorder: A Meta-analysis. Clin Psychopharmacol Neurosci. 2020;18(2):203-13. (doi: 10.9758/cpn.2020.18.2.203).

18. Stachon AC, Souza CD. Anxiety Disorders and Perceptual Disturbances in Adolescents with 22q11.2 Deletion Syndrome Treated with SSRI: A Case Series. J Can Acad Child Adolesc Psychiatry. 2011;20(4):305-10.

19. Massera D, Xu S, Walker MD, Valderrábano RJ, Mukamal $\mathrm{KJ}, \mathrm{Ix} \mathrm{JH}$, et al. Biochemical markers of bone turnover and risk of incident hip fracture in older women: the Cardiovascular Health Study. Osteoporos Int. 2019;30(9):1755-65. (doi: 10.1007/s00198-019-05043-1).

20. Gold PW, Gabry KE, Yasuda MR, Chrousos GP. Divergent endocrine abnormalities in melancholic and atypical depression: clinical and pathophysiologic implications. Endocrinol Metab Clin North Am 2002;31:37-62. (doi: 10.1016/S0889-8529(01)00022-6).

21. Kadriu B, Gold PW, Luckenbaugh DA, Lener MS, Ballard ED, Niciu MJ, et al. Acute ketamine administration corrects abnormal inflammatory bone markers in major depressive disorder. Mol Psychiatry. 2018;23(7):1626-31. (doi: 10.1038/mp.2017.109).

22. Trombetti A, Christ ER, Henzen C, Gold G, Brändle M, Herrmann FR, et al. Clinical presentation and management of patients with primary hyperparathyroidism of the Swiss Primary Hyperparathyroidism Cohort: a focus on neurobehavioral and cognitive symptoms. J Endocrinol Invest. 2016;39(5):567-76. (doi: 10.1007/s40618-015-0423-3).

23. McKeown R, Ellard DR, Rabiu AR, Karasouli E, Kearney RS. A systematic review of the measurement properties of patient reported outcome measures used for adults with an ankle fracture. J Patient Rep Outcomes. 2019;3(1):70. (doi: 10.1186/s41687-019-0159-5).

24. Davidson MR. Pharmacotherapeutics for osteoporosis prevention and treatment. J Midwifery Womens Health 2003;48:3952. (doi: 10.1016/S1526-9523(02)00359-8).

25. Coppen A, Bolander-Gouaille C. Treatment of depression: time to consider folic acid and vitamin B12. J Psychopharmacol 2005;19:59-65. (doi: 10.1177/0269881105048899). 
26. Kadriu B, Gold PW, Luckenbaugh DA, Lener MS, Ballard ED, Niciu MJ, et al. Acute ketamine administration corrects abnormal inflammatory bone markers in major depressive disorder. Mol Psychiatry. 2018;23(7):1626-31. (doi: 10.1038/mp.2017.109).

27. Bağış S. Clinical findings in osteoporosis, diagnosis and differential diagnosis. Mersin University Journal of Medicine 2002;1:83-8.

28. Demet D, Telci Fi, Dilbaz N, Okay IT. Psychotic disorder due to vitamin B12 deficiency. Bulletin of Clinical Psychopharmacology 2006;16:109-13.
29. Lerner V, Kanevsky M, Dwolatzky T, Rouach T, Kamin R, Miodownik C. Vitamin B 12 and Folate serum levels in newly admitted psychiatric patients. Clinical Nutrition 2006;25:60-7. (doi: 10.1016/j.clnu.2005.08.014).

30. Owiredu WKBA, Brenya PK, Osei Y, Laing EF, Okrah CO, Obirikorang $C$, et al. Evaluation of serum iron overload, AST:ALT ratio and log10ferritin:AST ratio among schizophrenia patients in the Kumasi Metropolis, Ghana: a case-control study. BMC Res Notes. 2019;12(1):802. (doi: 10.1186/s13104-019-4847-2). 